\title{
Psicopedagogía en Costa Rica: antecedentes y práctica
}

\author{
María Isabel Navarro-Guillén* \\ https://orcid.org/0000-0003-4172-1181
}

Recibido: 07 de mayo de 2020 • Aceptado: 24 de julio de 2020

\section{Resumen}

En este ensayo se realiza un análisis de la psicopedagogía en el contexto costarricense a partir de una exhaustiva revisión bibliográfica. Se resalta el papel protagónico de las universidades, las cuales forjaron el camino para el desarrollo de la psicopedagogía por medio de su intervención investigativa y formativa en el país. El creciente interés por mejorar los procesos de aprendizaje y la investigación educativa son acontecimientos significativos que impulsaron la institucionalización de la psicopedagogía como especialidad universitaria. No es hasta 1998, cuando surge como profesión, que se otorgan los primeros diplomas en licenciatura en psicopedagogía en la Universidad Nacional de Costa Rica. Actualmente, el campo psicopedagógico enfrenta desafíos como la consolidación epistemológica, la delimitación de las funciones profesionales y el aumento de los escenarios de ejercicio profesional.

Palabras clave: Epistemología, desarrollo profesional, institucionalización académica.

\footnotetext{
* Magister Scientiae en Psicopedagogía de la Universidad Estatal a Distancia (UNED), de Costa Rica. Licenciada en Educación Especial de la Universidad de Costa Rica (UCR), de Costa Rica. Tiene estudios en Sociología de la UCR. Psicopedagoga en privado. Correo: didaxiscr@gmail.com
} 


\title{
Psychopedagogy in Costa Rica: history and practice
}

\begin{abstract}
In this essay, an analysis of psychopedagogy in the Costa Rican context, drawn from an extensive bibliographic review, is carried out. The leading role of the universities is highlighted, as they forged the way for the development of psychopedagogy by way of their investigative and formative intervention in the country. The growing interest to improve learning processes and educational research are significant happenings which promoted the institutionalization of psychopedagogy as a university major. It wasn't until 1998, after this career arose, that the first degrees in psychopedagogy from the National University of Costa Rica were awarded. Currently, the area of psychopedagogy faces challenges such as its epistemological consolidation, demarcation of professional duties, and the increase in scenarios of professional practice in this area.
\end{abstract}

Key words: Epistemology, professional development, academic institutionalization.

\section{Psychopédagogie au Costa Rica: antécédents et pratique \\ Résumé}

Cet essai analyse la psychopédagogie dans le contexte costaricien à partir d'une révision bibliographique. Il met au premier plan le rôle des universités, lesquelles ont forgé le chemin pour le développement de cette discipline moyennant leur intervention formative et de recherche éducative dans le pays. L'intérêt grandissant pour améliorer les processus d'apprentissage et la recherche éducative sont des facteurs qui ont contribué à l'institutionnalisation de la psychopédagogie comme spécialité universitaire. Ce n'est qu'en 1998 quand la psychopédagogie émerge en tant que profession, lorsque les premiers diplômes en «licenciatura » (grade universitaire au Costa Rica, après la License) en psychopédagogie de l'Université Nationale du Costa Rica sont décernés. Actuellement, le domaine psychopédagogique relève des défis tels que la consolidation épistémologique, la délimitation des fonctions professionnelles et l'augmentation des scénarios d'exercice professionnel.

Mots-clés: Épistémologie, développement professionnel, institutionnalisation académique. 


\section{Introducción}

El presente ensayo se desarrolló desde un proceso de selección, consulta y análisis de diferentes fuentes, tales como revistas científicas, trabajos finales de graduación y libros, entre otros. A raíz del rastreo bibliográfico realizado, se evidenció la escasez de reseñas sobre el desarrollo profesional y la génesis de la psicopedagogía en Costa Rica. La mayor parte de las producciones investigativas en este campo se han orientado a temáticas como la escolarización y la intervención de problemas de aprendizaje.

Estos hallazgos respaldan la importancia de ampliar los escenarios investigativos para profundizar en la comprensión de la psicopedagogía. Es por esta razón que se revisará el camino que ha recorrido, es decir, los principales acontecimientos y los actores que participaron en acciones que dieron paso a su desarrollo. Se tratará, además, algunos aspectos actuales de la psicopedagogía entre ellos la complejidad epistemológica del campo, la delimitación de las prácticas profesionales y los ámbitos de ejercicio profesional.

\section{Antecedentes internacionales: desarrollo de la psicopedagogía}

La psicopedagogía surgió de la confluencia de dos tradiciones disciplinares: la psicología y la pedagogía. Con el pasar del tiempo, se trascendió de la aplicación de los conocimientos derivados de estas disciplinas a nutrirse de todas las ciencias que estudian a los seres humanos y a la sociedad de una forma directa e indirecta ${ }^{1}$, desplegando nuevos y más complejos saberes sobre la persona en situación de aprendizaje, interviniendo los procesos de construcción de conocimientos individuales y organizacionales, en las diferentes etapas del ciclo vital y en los diversos contextos de desarrollo.

El término psicopedagogía es relativamente reciente, aparece por primera vez como psycho-pédagogie en una publicación en 1908, en París, Francia, asociado a técnicas psicométricas destinadas a la clasificación de los escolares ${ }^{2}$. Poco a poco se fue normalizando el concepto y, junto con este, aparecieron otros derivados como psicopedagógico y psicopedagogo. Sin embargo, es importante señalar que las aportaciones teóricas y prácticas que prepararon el terreno para el desarrollo de la psicopedagogía toman fuerza desde el siglo XIX. Es en este periodo donde se da una proliferación de estudios, así como un aumento en la creación de instituciones para comprender y atender la psicopatología infantil, lo que resulta en una creciente incorporación de la psicología en las prácticas educativas.

${ }^{1}$ Emilio Ortiz y María de los Ángeles Mariño, «Una comprensión epistemológica de la psicopedagogía», Revista de Epistemología de Ciencias Sociales 49 (2014): 25, acceso: 25 de noviembre, 2020, https://scielo.conicyt.cl/scielo.php?pid=S0717554X2014000100003\&script=sci_arttext

${ }^{2}$ Rafael Bisquerra y Ángel C. Moreu, «Los orígenes de la psicopedagogía: el concepto y el término», REOP-Revista Española de Orientación y Psicopedagogía, n. ${ }^{\circ} 13$ (2002): 23, acceso: 25 de noviembre, 2020, http://revistas.uned.es/index.php/reop/article/viewFile/11583/pdf 
La necesidad de integrar el fundamento psicológico y experimental a la educación impulsó una serie de investigaciones en diferentes países. Algunos de los precursores teóricos que fortalecieron la interrelación e interdisciplinariedad de la psicología y la pedagogía fueron los estadounidenses W. James (1842-1910), J. Watson (18711958) y E. Thorndike (1874-1946), el suizo E. Claparede (1873-1940), los franceses A. Binet (1857-1911) y T. Simón (1858-1918) y el alemán K. Lewin (1890-1947). En el caso de los países latinoamericanos, se desarrollaron numerosos fenómenos psicológicos que fueron aplicados de manera directa al campo educativo, pero se hace difícil precisar los precursores que destacaron en la región, al constituirse en el terreno de muchos y patrimonio exclusivo de nadie ${ }^{3}$.

\section{Antecedentes nacionales}

La incorporación de prácticas psicológicas en los procesos educativos se fue extendiendo a diferentes países. En Costa Rica se inició con el despliegue de iniciativas que dieron paso a intervenciones pedagógicas más especializadas para atender nuevos servicios educativos. En 1939 surgió la educación especial con la apertura de la escuela Fernando Centeno Güell, encargada de brindar una atención más individualizada a niños considerados con retardo mental. En 1974 se crean las aulas diferenciadas, llamadas luego aulas integradas; en 1975 aparecieron los servicios de terapia de lenguaje; en 1977 los servicios de problemas de aprendizaje; en 1983 en trastornos emocionales y de conducta y, en esta misma década, se abren los programas de taller prevocacional para III ciclo en colegios técnicos ${ }^{4}$.

Estos nuevos servicios educativos surgen con el respaldo de cambios en la legislación; tal es el caso de la Ley Fundamental de Educación $N^{\circ} 2160$ creada en 1957, este, en el artículo 27, establece que la educación especial es la educación de los niños y adolescentes cuyas características físicas, mentales, emocionales o sociales se apartan del tipo normal, con el objeto de favorecer el desarrollo de sus capacidades y su incorporación a la sociedad como elementos útiles. Desde esta concepción, la educación especial se concibió como un sistema paralelo al sistema regular, dividiendo a la población estudiantil en dos grandes grupos. Esto se convirtió en un problema en el momento de establecer límites entre lo que se consideraba «normalidad» $\mathrm{y}$ «anormalidad». ¿Cómo determinar el grado de «retardo mental» que supera las posibilidades del buen educador ordinario? ¿Cuáles son los «trastornos emocionales» que justifican toda una organización especializada para atenderlos $?^{5}$ Estas y otras inquietudes favorecieron la incorporación de la psicología experimental y, en especial, de la psicometría en los entornos educativos para disponer de instrumentos y procedimientos que facilitaran la medición de distintos aspectos del aprendizaje y, de esta forma, agrupar a los estudiantes con el propósito de ofrecerles una atención acorde con sus necesidades. De esta manera, la educación especial, poco a poco, se transformó en el escenario educativo para las intervenciones psicológicas.

${ }^{3}$ Emilio Ortiz y María de los Ángeles Mariño, «Una comprensión epistemológica de la psicopedagogía»..., 24.

${ }^{4}$ Ministerio de Educación Pública, La educación especial y el mejoramiento de su gestión (San José: MEP, 1997), 4.

${ }^{5}$ Ministerio de Educación Pública, Oferta y demanda de la educación especial en Costa Rica (San José: MEP, 1989), 2. 
Estas intervenciones demandaron una formación más especializada y un número mayor de profesionales para atender los servicios. Inicialmente la cantidad de profesionales en educación especial no cubría la demanda a nivel nacional y no todos los profesionales contaban con la preparación requerida para atender los diferentes grupos de estudiantes. El estudio «Oferta y demanda de la educación especial en Costa Rica», realizado por el Ministerio de Educación Pública (MEP), mostró que la cantidad de niños y jóvenes atendidos en educación especial aumentó exponencialmente: en 1980 se registró 3233, en 1989 la cifra fue de 8012. En este periodo se formaban profesionales en educación especial en la Universidad de Costa Rica (UCR), con énfasis en problemas de aprendizaje, retardo mental, deficiencia visual, trastornos de comunicación y trastornos emocionales; mientras que en la Universidad Nacional (UNA) solo se formaban en ciencias de la educación con énfasis en problemas del aprendizaje. No existía una carrera que formara docentes en educación prevocacional, por lo que este servicio fue atendido por profesores de colegios técnico-profesionales. Además, la carencia de docentes en educación especial promovió que el personal docente de I y II ciclo de educación regular atendiera en gran parte las aulas recurso y las aulas diferenciadas ${ }^{6}$.

En este contexto se hace evidente el esfuerzo por aumentar la cobertura educativa, de manera que toda la niñez costarricense tuviera la posibilidad de acceder a la educación; pero, con la implementación de esta iniciativa, se vislumbró que el sistema educativo público no contaba con las condiciones necesarias para atender, de manera apropiada, a la diversidad que caracteriza al estudiantado. Esto genera una preocupación en las diferentes instituciones educativas, las cuales se dan a la tarea de promover medidas para fortalecer la educación y los servicios de educación especial emergentes. Una acción que busca garantizar la optimización educativa se da desde el MEP, al tratar de enriquecer los procesos de aprendizaje por medio de los aportes de la psicología. En 1982, el MEP establece la Dirección General de Psicopedagogía que inicialmente estaba integrada por los departamentos de educación especial, orientación, educación para la motivación y educación preescolar$^{7}$. Sus funciones consistían en aportar criterios, recursos y resultados de investigaciones sobre aspectos psicológicos que influían en la motivación, el aprendizaje, el desarrollo vocacional y la evaluación, así como de determinantes socioculturales del aprendizaje y la orientación profesional, además de factores del clima institucional y sobre las características de organización y participación estudiantil.

No obstante, los resultados de los estudios realizados por el MEP mostraron aspectos desalentadores con respecto al papel de esta asesoría, ya que se evidenció que no fue capaz de desarrollar un marco conceptual integrador ni su función se transformó en la sumatoria de las funciones generales de sus departamentos. Además, de la falta de correlación entre sus secciones, se presentaron constantes reajustes en la conformación de estos, lo que implicó cambios en los proyectos, objetivos y en el personal. Es por este motivo que en 1987 se propone el cambio de Asesoría General

\footnotetext{
${ }^{6}$ Ministerio de Educación Pública, Oferta y demanda de la educación especial en Costa Rica, 5-6.

${ }^{7}$ Ministerio de Educación Pública, Boletín de supervisión, junio, número 10 (San José: MEP, 1982), 11.

ESPIGA] @(1) @() Año 20, N. o 40: 191-209, julio - diciembre 2020 
de Psicopedagogía por el de Asesoría General de Orientación Estudiantil, lo que implicó un cambio en los departamentos que lo integraban y en sus funciones ${ }^{8}$.

Otra iniciativa que se impulsó en favor de la educación nacional y, sobre todo de los servicios de educación especial, la emprenden las universidades públicas mediante la mejora de la preparación del personal docente y la incorporación de los aportes de la psicología al ámbito educativo. Para esto, en 1992, la UCR, la UNA y la Universidad Estatal a Distancia (UNED) desarrollaron una propuesta de formación de docentes en educación especial. En el modelo del plan de estudios, proponen un curso de procesos de aprendizaje para estudiar, analizar y discutir los procesos mentales, cognitivos y ambientales necesarios para que se produzca el aprendizaje. También, consideraron en su plan, un curso de estrategias metodológicas para analizar los aportes de la psicología genética a la educación especial ${ }^{9}$. Dichas propuestas acentúan la relevancia de fortalecer el vínculo entre la disciplina de la psicología y la pedagogía para el mejoramiento de la intervención de los profesionales de educación especial en los diferentes ambientes de aprendizaje.

\section{Investigación educativa y su contribución al desarrollo de la psicopedagogía}

Las universidades públicas, además de destacarse en la formación profesional, se distinguieron en el campo investigativo. La UNA, en la década de 1980, por medio del Departamento de Psicología, desarrolló talleres, entrevistas y observaciones; estas acciones permitieron reconocer las principales problemáticas que afectaban las prácticas educativas, como la falta de recursos del MEP para atender la necesidad de capacitación, la ausencia de un modelo teórico-práctico de capacitación en el país y la escasez de capacitaciones de las universidades para actualizar a sus egresados. A raíz de esta situación, en 1987, el Departamento de Psicología justifica un proyecto permanente de capacitación a los docentes en el área psicopedagógica ${ }^{10}$.

La UCR, por su parte, en su labor de promoción de la investigación y la acción social, inauguró el Instituto de Investigación para el Mejoramiento de la Educación Costarricense (IIMEC), conocido en la actualidad como Instituto de Investigación en Educación (INIE) en 1980; de este Instituto, emergieron importantes proyectos como el Programa de Investigación en Epistemología Genética y Educación, del que se desprenden valiosos estudios. En educación preescolar sobresale la investigación: «Estandarización y desarrollo de normas costarricenses para la prueba selectiva del desarrollo de Denver», que da como resultado la creación de una herramienta ajustada a las características contextuales, lo que facilitó la detección de problemas

\footnotetext{
${ }^{8}$ Ministerio de Educación Pública, Situación actual y políticas de educación especial (San José: MEP,1987), 4-22.

${ }^{9}$ Consejo Nacional de Rectores, Propuesta de las instituciones de educación superior estatal: Universidad de Costa Rica, Universidad Nacional, Universidad Estatal a Distancia, para la formación de educadores en educación especial (San José,1992), 10-12.

${ }_{10}$ Zaira Escamilla, Ana Patricia Lizano y Stella Villegas, «Una respuesta de capacitación a maestros», Revista ABRA, n. ${ }^{\circ} 11$ (15-16, 1991):147, acceso: 25 de noviembre, 2020, https://www.revistas.una.ac.cr/index.php/abra/article/view/4419
}

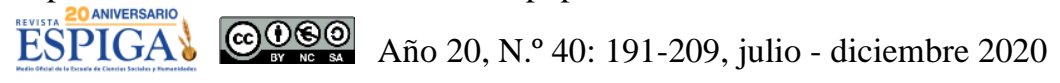


en el desarrollo y la intervención oportuna ${ }^{11}$. De igual forma, el «Programa experimental de niños en edad preescolar en familia. Plan piloto: Reapertura y desarrollo del programa de Hogares de Cuidado Diario», se dirigió al mejoramiento de la atención integral de la niñez en edad preescolar, en los objetivos, las autoras mencionan la necesidad de proporcionar actividades y estímulos psicopedagógicos a esta población para conseguir una atención integral ${ }^{12}$.

Entre los estudios psicogenéticos en educación primaria se encuentra: «El enfoque constructivo y el aprendizaje de la lectura», realizado en 1985, donde se resaltan los niveles psicogenéticos por los que pasa la niñez en el aprendizaje de la lectura y escritura, y cómo se encuentra ligado no solo a aspectos sensoriales y motores, sino que depende también de factores cognoscitivos ${ }^{13}$. Ese mismo año se publica: «El aprendizaje de la aritmética elemental por medio de juegos», que ofrece una descripción de actividades lúdicas a los educadores para abordar la enseñanza de nociones aritméticas, inspiradas en los estudios de desarrollo mental en la niñez realizados por el psicólogo suizo Jean Piaget ${ }^{14}$. En 1987, Aida Mainieri y Zaira Méndez presentan «Estudio psicogenético de la adición y la sustracción de una muestra de alumnos de primer año escolar de la región de Turrialba», en el que se evidenció que el personal docente promedio del país desconocía las características de la evolución psicopedagógica, conocimiento necesario para proporcionar actividades realmente estimulantes de acuerdo con el nivel en que se encontraba el estudiantado. Otro hallazgo de relevancia se da al comparar los resultados del pretest y del postest, ya que comprobó una evolución general de los estudiantes de niveles inferiores a niveles superiores del desarrollo. Esto demostró los efectos positivos de la implementación de prácticas educativas con un enfoque psicogenético ${ }^{15}$.

Se suma a estas investigaciones la realizada por la UCR y el Consejo Nacional de Investigaciones Científicas y Tecnológicas (CONICIT), llamada «Desarrollo del pensamiento formal en estudiantes de enseñanza secundaria del área metropolitana de San José». En sus resultados se sugiere la necesidad de crear un centro de investigación especializado en psicología y epistemología genética, donde prevalezca la colaboración interdisciplinaria para integrar sistemáticamente el trabajo de investigación en un campo que ofrece tantas posibilidades y que conecta con los problemas inmediatos que afectan el proceso educativo ${ }^{16}$.

${ }^{11}$ Douglas Howard y Marisol Nieto, Estandarización y desarrollo de normas costarricenses para la prueba selectiva del desarrollo de Denver (San José: IIMEC, 1981), 13.

12 Janina Bonilla Pignataro y Marta Eugenia Odio, Programa experimental de niños en edad pre-escolar en familia. Plan piloto: Reapertura y desarrollo del programa de Hogares de Cuidado Diario (San José: IIMEC,1985), 11.

${ }^{13}$ Aida Mainieri Hidalgo y Zaira Méndez, El enfoque constructivo y el aprendizaje de la lectura (San José: IIMEC, 1985), 4.

${ }^{14}$ Zaira Méndez Barrantes y Zulay Pereira Pérez, El aprendizaje de la aritmética elemental por medio de juegos (San José: IIMEC, 1985), 2.

${ }^{15}$ Aida Mainieri Hidalgo y Zaira Méndez, Estudio psicogenético de la adición y la sustracción de una muestra de alumnos de primer año escolar de la región de Turrialba (San José: IIMEC, 1987), 24-29.

16 Carmen Chaves, Ana Cecilia Escalante y Zaira Méndez, Desarrollo del pensamiento formal en estudiantes de enseñanza secundaria del área metropolitana de San José (San José: EUNED, 1983), 134. 
La labor de estas instituciones marca un momento crucial para la aplicación de los aportes de la psicología en los procesos educativos, al mismo tiempo que se fortalece la integración de estas disciplinas. Con sus estudios, evidencian la necesidad de realizar modificaciones en la formación docente, en los planes de estudio y en la metodología educativa, así como la importancia de conocer la psiquis infantil y su relación con el aprendizaje para mejorar las prácticas educativas. Esto impulsó el interés por desarrollar el campo de la psicopedagogía como especialidad.

\section{Institucionalización académica de la psicopedagogía}

En Costa Rica, la psicopedagogía, como especialidad universitaria, nace en la UNA. En 1995, desde el Consejo Nacional de Rectores (CONARE), se aprobó la solicitud de la licenciatura en psicopedagogía. En el documento de solicitud de aprobación de la licenciatura, la UNA señaló la contribución del Departamento de Psicología en la formación de educadores, resaltando que el departamento contaba con amplia experiencia para comprender el lugar que ocupaba la psicología en el ámbito pedagógico y los aportes que podía brindar a la educación costarricense.

Entre los aportes, mencionaron: modelos curriculares de la educación, procesos cognoscitivos, teorías del aprendizaje, teorías de la instrucción, relación pedagógica, desarrollo humano, procesos grupales, investigación en el aula, entre otros. La licenciatura en psicopedagogía de la UNA tomó como punto de partida al sujeto cognoscente como sujeto de estudio, sin dejar de lado el contexto en el cual lleva a cabo su práctica educativa. Los enfoques metodológicos se orientaron a que tanto docentes como estudiantes construyeran conocimientos en conjunto, además de que estos aprendizajes fueran significativos y trasferibles a la realidad, al seguir los principios de autores como Jerome Bruner y David Ausubel.

Esta licenciatura se configuró como una carrera de especialización y estaba dirigida a docentes y psicólogos con grado de bachiller. Se consideró que las personas interesadas en ingresar a la licenciatura serían aquellas que trabajaban y deseaban mejorar su conocimiento sobre los temas de esta carrera ${ }^{17}$. A la luz de estas precisiones, la psicopedagogía se originó ligada con el proceso educativo y con la optimización de las prácticas pedagógicas, a diferencia de otros países donde el quehacer psicopedagógico inició como una especialización en la evaluación y tratamiento de problemas de aprendizaje. En la licenciatura de psicopedagogía de la UNA, la formación profesional se dirigió al mejoramiento de los procesos de enseñanza y a la potenciación del aprendizaje en beneficio de toda la comunidad estudiantil y no de un grupo específico de personas.

En 1998, la UNA hizo entrega de los dos primeros diplomas de licenciatura en psicopedagogía ${ }^{18}$ y, hasta el 2004, se registró un total de 96 diplomas otorgados por dicha universidad en este grado. Con el tiempo, más universidades se interesan por incorporar la carrera de psicopedagogía en grado bachiller, licenciatura y maestría.

\footnotetext{
${ }^{17}$ Consejo Nacional de Rectores, Dictamen sobre la propuesta de creación de la licenciatura en psicopedagogía en la Universidad Nacional (San José: CONARE-OPES, 1995), 2-10.

${ }^{18}$ Consejo Nacional de Rectores, Estadística de los diplomas otorgados por las instituciones de educación superior universitaria de Costa Rica, 1990-2000 (San José: CONARE-OPES, 2001).
}

ESPIGA] @(1)(9) Año 20, N. 40 : 191-209, julio - diciembre 2020 
De acuerdo con datos publicados por el CONARE, en estadística de los diplomas otorgados por las instituciones de educación superior universitaria de Costa Rica, solo la Universidad Autónoma de Centro América (UACA) implementa el bachillerato y la licenciatura en psicopedagogía y, hasta el 2018, esta universidad otorgó 43 diplomas en bachillerato y 30 diplomas en licenciatura en psicopedagogía.

A nivel nacional, la mayor cantidad de títulos en psicopedagogía corresponde al grado de maestría. En la tabla que se presenta a continuación, se muestra el número de diplomas dado desde el 2000 hasta el 2018 en el grado de maestría por las siguientes universidades: Florencio del Castillo (UCA), Hispanoamericana (UH), Juan Pablo II (UJP II), Latina de Costa Rica (ULatina), Latinoamericana de Ciencia y Tecnología (ULACIT), La Salle (UlaSalle), Nacional (UNA), Estatal a Distancia (UNED) y la de San José (USJ).

Tabla 1 Cantidad de diplomas de maestría en psicopedagogía otorgados por universidades en Costa Rica del 2000 al 2018

\begin{tabular}{|l|c|c|c|c|c|c|c|c|c|c|}
\hline Año & UCA & UH & $\begin{array}{c}\text { UJP } \\
\text { II }\end{array}$ & ULatina & ULACIT & UlaSalle & UNA & UNED & USJ & Total \\
\hline 2000 & - & - & - & - & - & 22 & - & 25 & - & 47 \\
\hline 2001 & - & - & - & - & - & 36 & - & 33 & - & 69 \\
\hline 2002 & - & - & - & - & - & 51 & - & 16 & - & 67 \\
\hline 2003 & - & - & - & - & - & 36 & - & 10 & - & 46 \\
\hline 2004 & - & - & 5 & - & - & 43 & - & 16 & - & 64 \\
\hline 2005 & - & - & - & - & 12 & 38 & - & 50 & - & 100 \\
\hline 2006 & - & - & - & 94 & 14 & 28 & - & 25 & 16 & 177 \\
\hline 2007 & 10 & - & - & 104 & 14 & 29 & 22 & 35 & 32 & 246 \\
\hline 2008 & 25 & - & - & 70 & 6 & 17 & 18 & 10 & 24 & 170 \\
\hline 2009 & 28 & - & - & 112 & 3 & 20 & - & 53 & 40 & 256 \\
\hline 2010 & 36 & - & - & 48 & 3 & 45 & - & 45 & 32 & 209 \\
\hline 2011 & 55 & 7 & - & 53 & - & 38 & - & 41 & 38 & 232 \\
\hline 2012 & 40 & 33 & - & 43 & - & 39 & - & 31 & 23 & 209 \\
\hline 2013 & 30 & 25 & - & 39 & - & 16 & 7 & 33 & 26 & 176 \\
\hline 2014 & 40 & 29 & - & 54 & 1 & 23 & 9 & 23 & 13 & 192 \\
\hline 2015 & 26 & 8 & - & 40 & 2 & 16 & - & 15 & 13 & 120 \\
\hline 2016 & 11 & 33 & - & 34 & 2 & 14 & - & 17 & 22 & 133 \\
\hline 2017 & 20 & 26 & - & 25 & 2 & 16 & - & 11 & 23 & 123 \\
\hline 2018 & 20 & - & - & 67 & - & 9 & - & 14 & 22 & 132 \\
\hline Total & 341 & 161 & 5 & 783 & 59 & 536 & 56 & 503 & 324 & 2768 \\
\hline
\end{tabular}

Fuente: Elaboración propia de la autora (2020) a partir de las estadísticas de diplomas otorgados por las instituciones de educación superior universitaria de Costa Rica, CONARE. 
Los datos de la tabla muestran que una cantidad significativa de personas opta por la maestría en psicopedagogía en las diversas universidades del país, tanto públicas como privadas. Lo anterior respalda la relevancia de realizar un análisis sobre su epistemología en el contexto actual y sobre el ejercicio profesional de sus egresados.

\section{Epistemología de la psicopedagogía}

El aumento de profesionales en el área de psicopedagogía exige atender los direccionamientos teóricos para dilucidar el saber que consolida al campo psicopedagógico. Con respecto al estatus científico de la psicopedagogía, se presentan criterios divergentes entre los investigadores; algunos la reconocen como una disciplina que registra una corta historia ${ }^{19}$, mientras que otros son del criterio de que, tanto como disciplina científica como de formación para el ejercicio profesional, goza de carácter autónomo desde hace más de un siglo, emergiendo como disciplina científica a inicios del siglo $\mathrm{XX}^{20}$.

Por otra parte, existen autores que afirman que no se puede concebir a la psicopedagogía como una disciplina científica, debido a problemáticas teóricoepistemológicas. Argumentan que la psicopedagogía no es un campo de investigación consolidado, por lo que tiene una estructura de conocimiento débil y de baja intensidad que no le permite explicitar ni integrar su objeto de estudio ni el repertorio metodológico; asimismo, las determinaciones del campo han sido incapaces de asumir una posición concreta respecto de su base epistemológica ${ }^{21}$. Por esta razón, se afirma que actualmente se encuentra en vías de su constitución disciplinar, de consolidar su identidad y de construir marcos conceptuales y metodológicos que emanen de la especificidad de su propia práctica ${ }^{22}$.

De igual forma, existe un debate con respecto a los límites del campo psicopedagógico. Algunos autores coinciden en que se encuentra en construcción y, hoy por hoy, no cuenta con una delimitación clara. La ampliación de funciones de la intervención, así como de los ámbitos de acción, ha creado una intersección difícil de diferenciar. La tarea de establecer límites precisos entre la psicología educacional, la psicopedagogía y la educación especial, entre otras, resulta compleja. Esta dificultad para establecer una delimitación de sus respectivas identidades reside en que recurren a bases teóricas comunes y tienen campos de aplicación convergentes,

${ }^{19}$ Elisa Azar, «Reflexiones sobre el campo psicopedagógico. La psicopedagogía escolar», Diálogos pedagógicos, . $^{\circ} 10$ (20-2012), 75, acceso: 25 de noviembre, 2020, http://revistas.bibdigital.ucc.edu.ar/index.php/dialogos/article/view/563

${ }^{20}$ José Tomás Bethencourt Benítez y Lidia Cabrera Pérez, «La psicopedagogía como ámbito científico-profesional», Electronic Journal of Research in Educational Psychology, n. ${ }^{\circ} 8$ (2, 2010): 904, acceso: 25 de noviembre, 2020, https://www.redalyc.org/pdf/2931/293122002021.pdf

21 Aldo Ocampo González, «En torno a la perturbación del objeto de la Psicopedagogía: tensiones analítico-metodológicas», En: Ensayos críticos sobre Psicopedagogía en Latinoamérica (Santiago de Chile: CELEI, 2018), 92-141, edición en PDF, acceso: 25 de noviembre, 2020, https://www.aacademica.org/aldo.ocampo.gonzalez/18

${ }^{22}$ Juan Antonio Artacho y Ana Clara Ventura, «Psicopedagogía e interdisciplina: reflexiones desde una perspectiva epistemológica», Forma Sociedad Editora; Aprendizaje, Hoy, 80 (62011), 12-13, acceso: 25 de noviembre, 2020, https://ri.conicet.gov.ar/handle/11336/14096 
lo que genera entre ellas una identidad parcialmente compartida ${ }^{23}$. Para establecer límites claros, se requiere construir una nueva disciplina que se defina a partir de su propio campo, para entablar el diálogo necesario con otras disciplinas que le demanda la complejidad de su objeto ${ }^{24}$.

Para superar estas imprecisiones, se propone reconocer el carácter fronterizo e intermediador del objeto de la psicopedagogía, es decir, la noción de «objeto fronterizo» que permite analizar la manera en que el fenómeno psicopedagógico es atravesado por una heterogeneidad de campos disciplinarios y discursivos, incorporando elementos y singularidades epistemológicas y objetos diversificados ${ }^{25}$. Se propone además, posicionar a la psicopedagogía como ámbito interdisciplinar, en el cual tienen que confluir o converger necesariamente la psicología de la educación, la orientación educativa, la educación especial, el diagnóstico o evaluación psicopedagógica y las bases didácticas para la intervención, entre otras ${ }^{26}$.

Asimismo, se debe considerar que la estructura de conocimiento de la psicopedagogía se caracteriza por plantear múltiples formas de conocer y consagrar una estructura teórica y metodológica abierta, por lo que su construcción epistemológica no puede cerrarse a través de las formas epistemológicas y metodológicas tradicionales ${ }^{27}$. Todas estas consideraciones merecen ser analizadas para implementar medidas y propuestas que permitan alcanzar un estatus disciplinar.

En el caso de Costa Rica, diferentes autores reconocen a la psicopedagogía como una disciplina científica; en la investigación de María Gabriela Rodas, se hace referencia a la psicopedagogía como una rama de la psicología aplicada a la educación que investiga problemas educacionales por medio de métodos y conceptos psicológicos, muchos de ellos creados para este propósito y que, agrega, la psicopedagogía cuenta con epistemología propia ${ }^{28}$. Por su parte, Paula María Castillo describe la psicopedagogía como la amalgama disciplinar entre la psicología y la pedagogía ${ }^{29}$. En el estudio de Silvia Méndez se acoge el concepto de psicopedagogía de Marina Müller, al entenderla como una disciplina cuyo objeto de estudio es el aprendizaje humano ${ }^{30}$. Laura Patricia Rivera emplea la definición de Henao, Ramírez y Ramírez, en la cual se describe a la psicopedagogía como la disciplina profesional en la que confluyen posturas teóricas, ideológicas, concepciones éticas de disciplinas como la psicología, la pedagogía, el trabajo social

${ }^{23}$ Luis Bravo, «Psicología educacional, psicopedagogía y educación especial», Revista II PSI Facultad de Psicología, n. ${ }^{\circ} 12$ (2-2009), 217-218, acceso: 25 de noviembre, 2020, http://repositorio.minedu.gob.pe/handle/123456789/2338

${ }^{24}$ Elisa Azar, «Reflexiones sobre...», 75.

${ }^{25}$ Aldo Ocampo González, «En torno..., 102-106.

${ }^{26}$ José Tomás Bethencourt Benítez y Lidia Cabrera Pérez. «La psicopedagogía..., 899.

${ }^{27}$ Aldo Ocampo González, «En torno..., 124.

${ }^{28}$ María Gabriela Rodas Agüero, «Rol actual del psicopedagogo como miembro de una estructura de apoyo externo a los centros educativos» (TFG para optar por el título de maestría, UNED, 2003), 29.

29 Paula María Castillo Castillo, «Análisis psicopedagógico desde el paradigma constructivista de una estrategia de aprendizaje a través de la plataforma virtual Schoology» (TFG para optar por el grado de maestría, UNED, 2014), 23.

30 Silvia Méndez Anchía, «Comprensión lectora y textos literarios: una propuesta psicopedagógica», Revista Educación 30 (1-2006), 143-144, acceso: 25 de noviembre, 2020, http://www.redalyc.org/articulo.oa?id=44030109

ESPIGA- @(1) (1) Añ 2020 
y la medicina, entre otras, centradas en el aprendizaje ${ }^{31}$. Estas publicaciones se limitan a la descripción conceptual. No se encuentran estudios a nivel nacional que se refieran en profundidad al marco conceptual y epistemológico que sustenta a la psicopedagogía como disciplina.

Partiendo de que los aportes teóricos anteriores se configuran ligados a la dimensión práctica, se hace necesario realizar un acercamiento al desarrollo profesional en este campo, no solo para conocer el contexto de aplicación de lo psicopedagógico, sino también para reflexionar sobre la delimitación de funciones de estos profesionales.

\section{Desarrollo profesional actual}

En Costa Rica no se encuentra una delimitación clara de las funciones profesionales de los psicopedagogos, puesto que no existe un marco legal ni un colegio profesional que determine y regule el ejercicio profesional, ético y competente de estos profesionales. A la fecha, se ha considerado el grado que sirvió de base para obtener la maestría en psicopedagogía para definir a cuál colegio pertenece una persona psicopedagoga. Ejemplo de ello es la Dirección General de Servicio Civil, rectora del empleo público en el Estado costarricense, que en la resolución DG-346-2005 incluye la maestría profesional en psicopedagogía como atinencia académica en especialidades como: divulgación educativa, equipos interdisciplinarios en el área funcional educativa e investigación educativa. Este organismo aclara que la maestría es aceptada para aquellas clases que requieran el grado de bachillerato o licenciatura, siempre y cuando la persona candidata, en la eventualidad de un nombramiento o ascenso, esté debidamente incorporado al colegio profesional respectivo.

Sin embargo, en la actualidad hay profesionales cuyo grado en bachillerato y licenciatura corresponde a psicopedagogía, por lo que no tienen la opción de pertenecer a un colegio profesional. Al no contar con un colegio profesional, no se les puede impedir el ejercicio de la psicopedagogía. De igual forma, los psicopedagogos que desarrollan su propia empresa y trabajan en consultorios privados pueden hacerlo sin necesidad de pertenecer a algún colegio profesional. Tanto los psicopedagogos que pertenecen como los que no pertenecen a un colegio profesional, se encuentran ejerciendo su profesión sin lineamientos claros sobre sus funciones.

En lo que respecta a las opciones laborales donde se pueden desempeñar los profesionales en psicopedagogía, se mencionan varias alternativas. En la propuesta de creación de la licenciatura en psicopedagogía de la UNA, se señala que la persona graduada de esta carrera podría trabajar en instituciones educativas de nivel preescolar, de primaria, de secundaria y de educación superior e instituciones formadoras o de capacitación informal ${ }^{32}$, al demostrar que inicialmente la formación de este profesional se orientaba a la escolarización. Siguiendo en esta línea, en el estudio de María Gabriela Rodas se menciona que el rol del profesional en

${ }^{31}$ Laura Patricia Rivera Alvarado, «Análisis psicopedagógico del servicio de orientación vocacional de un colegio privado de la provincia de San José», Revista Actualidades Investigativas en Educación, n. ${ }^{\circ} 14$ (3-2014), 10, acceso: 25 de noviembre, 2020, https://revistas.ucr.ac.cr/index.php/aie/article/view/16087/15529

32 Consejo Nacional de Rectores (CONARE), Dictamen sobre..., 11. 
psicopedagogía, como miembro de una estructura de apoyo externo a los centros educativos, se subdivide en tres funciones: especialista clínico, orientador de docentes y en su labor de apoyo a padres y madres de familia ${ }^{33}$.

Actualmente se consideran más espacios laborales para los profesionales de psicopedagogía, algunos de los campos que expone la UCA para las personas graduadas son: prácticas educativas escolares, servicios especializados de orientación educativa y psicopedagógica, centros específicos y servicios de educación especial, evaluación de programas, planificación y gestión educativa, investigación educativa, educación de adultos, centros de diagnóstico y geriatría, entre otros ${ }^{34}$.

La ULatina menciona los centros educativos públicos y privados, atención individual privada, organizaciones no gubernamentales (ONG), coordinación de equipos interdisciplinarios de atención a la población en riesgo social, empresas en las áreas relacionadas con el desarrollo del potencial humano, diseño de material gráfico o de software pedagógico para el desarrollo de las habilidades y destrezas del aprendizaje $^{35}$. La UlaSalle destaca: clínicas de problemas de aprendizaje, de adaptación de grupos, problemas de la tercera edad, de reeducación de adolescentes y de jóvenes y consejero en centros educativos de cualquier nivel ${ }^{36}$. Otros escenarios de trabajo que menciona la ULACIT son: centros de salud mental, hospitales, centros de atención temprana, profesor universitario en el área de la docencia y psicopedagogía, capacitador de personal técnico, administrativo y docente ${ }^{37}$.

Estos datos ponen en evidencia que el objetivo inicial de la psicopedagogía, de formar especialistas para mejorar sus prácticas profesionales y los procesos de aprendizaje, trascendió las fronteras de la educación formal y no formal para abrirse espacio en ámbitos empresariales, comunales y de salud, entre otros. Pero, aunque las universidades promueven que los profesionales en psicopedagogía en Costa Rica están en la capacidad de laborar en estos campos, lo cierto es que no existen muchos de estos puestos laborales. Por ejemplo, no se encuentran investigaciones, a nivel nacional, que evidencien la participación de profesionales en psicopedagogía en procesos de orientación vocacional ${ }^{38}$.

Asimismo, en el estudio de egresados de la maestría de psicopedagogía de la UNED, de 49 participantes solo una persona indicó que su trabajo principal era como psicopedagoga en un centro educativo privado. La mayor parte de los graduados

\footnotetext{
${ }^{33}$ María Gabriela Rodas Agüero, «Rol actual..., 27.

34 «Maestría profesional en psicopedagogía», Universidad Florencio del Castillo, UCA, acceso: 6 de agosto, 2020, https://www.uca.ac.cr/carreras/maestria-profesional-enpsicologia/

35 «Maestría en psicopedagogía», Universidad Latina, acceso: 6 de agosto, 2020, https://ulatina.ac.cr/wpcontent/uploads/2017/12/Programa_Psicopedagog\%C3\%ADa_SP_baja.pdf

36 «Maestría en psicopedagogía», Universidad La Salle, acceso: 7 de agosto, 2020, https://www.ulasalle.ac.cr/posgrados/

37 «Perfil ocupacional», Universidad Latinoamericana de Ciencia y Tecnología, acceso: 7 de agosto, 2020,

http://www.ulacit.ac.cr/carreras/seccion/perfil.php?career=6\&grade_id=5\&id=106

${ }^{38}$ Laura Patricia Rivera Alvarado, «Análisis psicopedagógico del servicio de orientación vocacional de un colegio privado de la provincia de San José»..., 3.

ESPIGA' @) (1) (2) Año 20, N. ${ }^{\circ} 40: 191-209$, julio - diciembre 2020 
consultados en esta investigación se dedicaban a la docencia y psicología ${ }^{39}$. Esta tendencia se puede explicar con los resultados del «Estudio de seguimiento de las personas graduadas del periodo 2000-2006 de once programas de posgrado de las universidades estatales», en los que incluyó a la maestría de psicopedagogía de la UNED. En términos generales, los participantes de este estudio indicaron que el estudiantado logra mejorar su práctica y la maestría tiene repercusiones en la parte económica, ya que significa asensos en las carreras profesionales ${ }^{40}$, lo que muestra que se elige este posgrado en muchas ocasiones para mejorar el ejercicio profesional en campos relacionados con la educación formal y para recibir una mayor remuneración económica.

Estas condiciones reafirman la concepción de que Latinoamérica, con excepción de Argentina, se caracteriza por formar a los profesionales en psicopedagogía para la escolarización ${ }^{41}$, lo que dificulta la presencia de este profesional en centros de salud, centros de atención a población en riesgo social, instituciones que atienden a personas de la tercera edad y empresas, entre otros. Las universidades que imparten psicopedagogía señalan que el sistema educativo formal es un entorno minoritario de ejercicio profesional; pero en la realidad, la práctica psicopedagógica sigue concentrándose en consultorios independientes e instituciones educativas privadas, lo que priva a un gran número de personas de recibir una atención integral de la más alta calidad. Esto se convierte en un desafío importante que debe ser atendido con prontitud y eficacia por parte de los formadores de los profesionales en psicopedagogía y los entes de diferentes instituciones, tanto públicas como privadas, para garantizar una preparación de los egresados de bachillerato, licenciatura y maestría en psicopedagogía acorde a las demandas laborales, a la vez que se impulsen más espacios para ejercer la profesión. Pero, para alcanzar este propósito, se requiere de la implementación de programas de seguimiento de los egresados y establecer un diálogo continuo con los entes empleadores para que se satisfagan las demandas y necesidades de todos los involucrados. En la actualidad, no hay evidencia de que estos lineamientos se cumplan, por lo que se hace urgente hacer un alto en el camino y evaluar hacia dónde se dirige la psicopedagogía con las medidas que se han implementado y valorar cuál es el punto al que se quiere llegar. Esto con el fin de plantear y desarrollar acciones que posibiliten un perfeccionamiento cada vez mayor del campo psicopedagógico que responda satisfactoriamente a las transformaciones y a los requerimientos de la sociedad costarricense.

\section{Consideraciones finales}

Los datos presentados anteriormente permiten constatar que la psicopedagogía en Costa Rica, como concepto y como acciones metodológicas, se empieza a generalizar desde la década de 1980. Como quehacer profesional, derivó de la

\footnotetext{
${ }^{39}$ María Isabel Navarro, «Análisis acerca del grado de satisfacción laboral del estudiantado graduado entre los años 2010 al 2015 de la maestría en psicopedagogía de la Universidad Estatal a Distancia, a partir del modelo de dos factores de Herzberg» (TFG para optar por el grado de maestría, UNED, 2015), 138.

${ }^{40}$ Consejo Nacional de Rectores, Estudio de seguimiento de las personas graduadas del periodo 2000 -2006 de once programas de posgrado de las universidades estatales (San José, Costa Rica: EUNED, 2009), 116.

${ }^{41}$ Aldo Ocampo González, «En torno..., 98. ESPIGA' @) (1) (2) Año 20, N. ${ }^{\circ} 40: 191-209$, julio - diciembre 2020 
necesidad de mejorar los procesos de aprendizaje en el ámbito escolar principalmente, constituyéndose en una especialización para optimizar la formación profesional y la intervención educativa.

La psicopedagogía a nivel nacional enfrenta importantes desafíos que deben ser atendidos. La historia ha demostrado la repercusión de la investigación en el desarrollo de la psicopedagogía, por lo que hoy más que nunca se deben desarrollar estudios que permitan debatir, comprender y mejorar la consolidación del espacio científico y profesional que le otorgue una identidad sólida al campo psicopedagógico en el escenario costarricense. Las universidades, especialmente las que imparten psicopedagogía, tienen la responsabilidad de participar activamente en la construcción de los conocimientos psicopedagógicos y generar los espacios requeridos para que, tanto el estudiantado como las personas graduadas, reflexionen sobre la psicopedagogía y sus desafíos. Y desde estas reflexiones desarrollen transformaciones que consoliden los fundamentos epistemológicos de la psicopedagogía y potencien su ejercicio profesional.

Finalmente se considera relevante la definición del perfil profesional de los psicopedagogos fuera del entorno escolar, para que las instancias gubernamentales y no gubernamentales fomenten la institucionalización de los servicios psicopedagógicos. Es relevante habilitar nuevos espacios de actuación para los profesionales de psicopedagogía y garantizar que los egresados cuentan con la formación requerida para ejercer en dichos puestos. De esta manera, las personas que necesitan los servicios psicopedagógicos podrán disponer de la atención que merecen. La estabilización de la actividad psicopedagógica en los centros educativos, en los centros de salud, en centros de atención a población en riesgo social, instituciones que atienden a personas de la tercera edad y en el sector empresarial, entre otros; es una gran oportunidad para beneficiar a todas las personas y para asegurar la calidad del servicio que prestan las diferentes entidades.

\section{Formato de citación según APA}

Navarro-Guillén, M. I. (2020). Psicopedagogía en Costa Rica: antecedentes y práctica. Revista Espiga, 20 (40), páginas 191-209

Formato de citación según Chicago-Deusto

Navarro-Guillén, María Isabel. «Psicopedagogía en Costa Rica: antecedentes y práctica». Revista Espiga 20, n. ${ }^{\circ} 40$ (julio-diciembre, 2020): páginas 191-209 


\section{Fuentes consultadas}

Artacho, Juan Antonio y Ana Clara Ventura. «Psicopedagogía e interdisciplina: reflexiones desde una perspectiva epistemológica». Forma Sociedad Editora; Aprendizaje, Hoy, n. ${ }^{\circ} 80$ (6-2011), 7-16. Acceso: 25 de noviembre, 2020. https://ri.conicet.gov.ar/handle/11336/14096

Asamblea Legislativa. Ley Fundamental de Educación 2160. San José, Costa Rica, 1957.

Azar, Elisa. «Reflexiones sobre el campo psicopedagógico. La psicopedagogía escolar». Diálogos pedagógicos, n. ${ }^{\circ}$ 10(20-2012), 74-98. Acceso: 25 de noviembre, 2020. http://revistas.bibdigital.ucc.edu.ar/index.php/dialogos/article/view/563

Baeza, Silvia. «Psicopedagogía: nuevos desafíos hoy... hacia las mejores prácticas del mañana». Revista Contextos de Educación, n. ${ }^{\circ} 12$ (2012). Acceso: 25 de noviembre, 2020.

http://www.enfermeria.hum.unrc.edu.ar/publicaciones/contextos/articulos/ vol12/pdfs/05-baeza.pdf

Bethencourt Benítez, José Tomás y Lidia Cabrera Pérez. «La psicopedagogía como ámbito científico-profesional». Electronic Journal of Research in Educational Psychology, n. ${ }^{\circ} 8$ (2-2010): 893-914. Acceso: 25 de noviembre, 2020. https://www.redalyc.org/pdf/2931/293122002021.pdf

Bisquerra, Rafael y Ángel C Moreu. «Los orígenes de la psicopedagogía: el concepto y el término». REOP-Revista Española de Orientación y Psicopedagogía, n. ${ }^{\circ} 13$ (2002): 17-29. Acceso: 25 de noviembre, 2020. http://revistas.uned.es/index.php/reop/article/viewFile/11583/pdf

Bravo, Luis. «Psicología educacional, psicopedagogía y educación especial». Revista II PSI Facultad de Psicología, n. ${ }^{\circ} 12$ (2-2009): 217-225. Acceso: 25 de noviembre, 2020. http://repositorio.minedu.gob.pe/handle/123456789/2338

Castillo Castillo, Paula María. «Análisis psicopedagógico desde el paradigma constructivista de una estrategia de aprendizaje a través de la plataforma virtual Schoology para favorecer la orientación vocacional de estudiantes del Colegio Marista de Alajuela». TFG para optar por el grado de maestría, UNED, 2014. Recuperado de http://repositorio.uned.ac.cr/reuned/handle/120809/1377

Chaves, Carmen, Ana Cecilia Escalante y Zaira Méndez. Desarrollo del pensamiento formal en estudiantes de enseñanza secundaria del área metropolitana de San José. San José: EUNED, 1983.

Consejo Nacional de Rectores. Propuesta de las instituciones de educación superior estatal: Universidad de Costa Rica, Universidad Nacional, Universidad Estatal a Distancia, para la formación de educadores en educación especial. San José: CONARE, 1992.

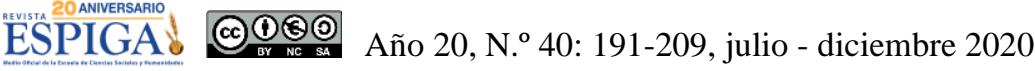


Consejo Nacional de Rectores. Dictamen sobre la propuesta de creación de la licenciatura en psicopedagogía en la Universidad Nacional. San José: CONARE-OPES, 1995.

Consejo Nacional de Rectores. Estadística de los diplomas otorgados por las instituciones de educación superior universitaria de Costa Rica, 1990-2000. San José: CONARE-OPES, 2001.

Consejo Nacional de Rectores. Estadística de los diplomas otorgados por las instituciones de educación superior universitaria de Costa Rica, 2001-2005. San José: CONARE-OPES, 2008a.

Consejo Nacional de Rectores. Estadística de los diplomas otorgados por las instituciones de educación superior universitaria de Costa Rica, 2006. San José: CONARE-OPES, 2008 b.

Consejo Nacional de Rectores. Estudio de seguimiento de las personas graduadas del periodo 2000 -2006 de once programas de posgrado de las universidades estatales. San José: EUNED, 2009.

Consejo Nacional de Rectores. Estadística de los diplomas otorgados por las instituciones de educación superior universitaria de Costa Rica, 2007 y 2008. San José: CONARE-OPES, 2010.

Consejo Nacional de Rectores. Estadística de los diplomas otorgados por las instituciones de educación superior universitaria de Costa Rica, 2009. San José: CONARE-OPES. 2012.

Consejo Nacional de Rectores. Estadística de los diplomas otorgados por las instituciones de educación superior universitaria de Costa Rica, 2010. San José: CONARE-OPES, 2015a.

Consejo Nacional de Rectores. Estadística de los diplomas otorgados por las instituciones de educación superior universitaria de Costa Rica, 2011. San José: CONARE-OPES, 2015.

Consejo Nacional de Rectores. Estadística de los diplomas otorgados por las instituciones de educación superior universitaria de Costa Rica, 2012. San José: CONARE-OPES. 2015c.

Consejo Nacional de Rectores. Estadística de los diplomas otorgados por las instituciones de educación superior universitaria de Costa Rica, 2013. San José: CONARE-OPES, 2017a.

Consejo Nacional de Rectores. Estadística de los diplomas otorgados por las instituciones de educación superior universitaria de Costa Rica, 2014. San José: CONARE-OPES, $2017 \mathrm{~b}$. 
Consejo Nacional de Rectores. Estadística de los diplomas otorgados por las instituciones de educación superior universitaria de Costa Rica, 2015. San José: CONARE-OPES, 2017c.

Consejo Nacional de Rectores. Estadística de los diplomas otorgados por las instituciones de educación superior universitaria de Costa Rica, 2016-2018. San José: CONARE-OPES (comunicación personal el viernes 13 de marzo del 2020 mediante correo electrónico).

Escamilla, Zaira, Ana Patricia Lizano y Stella Villegas. «Una respuesta de capacitación a maestros. Revista ABRA, n. ${ }^{\circ} 11$ (15-16-1991): 143-157. Acceso: $\quad 25 \quad$ de $\quad 2020$. https://www.revistas.una.ac.cr/index.php/abra/article/view/4419

Howard, Douglas y Marisol Nieto. Estandarización y desarrollo de normas costarricenses para la prueba selectiva del desarrollo de Denver. San José: IIMEC, 1981.

Mainieri Hidalgo, Aida y Zaira Méndez. El enfoque constructivo y el aprendizaje de la lectura. San José: IIMEC, 1985.

Mainieri Hidalgo, Aida y Zaira Méndez. Estudio psicogenético de la adición y la sustracción de una muestra de alumnos de primer año escolar de la región de Turrialba. San José: IIMEC, 1987.

Méndez Anchía, Silvia. «Comprensión lectora y textos literarios: una propuesta psicopedagógica». Revista Educación 30 (1-2006), 141-155. Acceso: 25 de noviembre, 2020. http://www.redalyc.org/articulo.oa?id=44030109

Méndez Barrantes, Zaira y Zulay Pereira Pérez. El aprendizaje de la aritmética elemental por medio de juegos. San José: IIMEC, 1985.

Ministerio de Educación Pública. Boletín de supervisión, junio, número 10. San José: MEP, 1982.

Ministerio de Educación Pública. Situación actual y políticas de educación especial. San José: MEP, 1987.

Ministerio de Educación Pública. Oferta y demanda de la educación especial en Costa Rica. San José: MEP, 1989.

Ministerio de Educación Pública. La educación especial y el mejoramiento de su gestión. San José: MEP, 1997.

Navarro, María Isabel. «Análisis acerca del grado de satisfacción laboral del estudiantado graduado entre los años 2010 al 2015 de la maestría en psicopedagogía de la Universidad Estatal a Distancia, a partir del modelo de dos factores de Herzberg». TFG para optar por el grado de maestría, UNED, 2015. 
Ocampo González, Aldo. «En torno a la perturbación del objeto de la Psicopedagogía: tensiones analítico-metodológicas». En: Ensayos críticos sobre Psicopedagogía en Latinoamérica. Chile: CELEI. 2018. 91-186. Acceso: $\quad 25 \quad$ de noviembre, 2020. https://www.aacademica.org/aldo.ocampo.gonzalez/18

Ortiz, Emilio. «La psicopedagogía como ciencia y profesión en el contexto iberoamericano». En: Ensayos críticos sobre Psicopedagogía en Latinoamérica. Chile: CELEI, 2018. 63-89. Acceso: 25 de noviembre, 2020. https://www.aacademica.org/aldo.ocampo.gonzalez/18

Ortiz, Emilio y María de los Ángeles Mariño. «Una comprensión epistemológica de la psicopedagogía». Revista de Epistemología de Ciencias Sociales, $\mathrm{n} .{ }^{\circ} 49$ (2014): 22-30. Acceso: 25 de noviembre, 2020. https://scielo.conicyt.cl/scielo.php?pid=S0717554X2014000100003\&script=sci_arttext

Rivera Alvarado, Laura Patricia. «Análisis psicopedagógico del servicio de orientación vocacional de un colegio privado de la provincia de San José». Revista Actualidades Investigativas en Educación, n. ${ }^{\circ} 14$ (3, 2014). Acceso: 25 de noviembre, 2020. https://revistas.ucr.ac.cr/index.php/aie/article/view/16087/15529

Rodas Agüero, María Gabriela. «Rol actual del psicopedagogo como miembro de una estructura de apoyo externo a los centros educativos». TFG para optar por el título de maestría, UNED, 2003. Acceso: 25 de noviembre, 2020. https://repositorio.uned.ac.cr/handle/120809/931?locale-attribute=en

Universidad Florencio del Castillo, UCA. «Maestría profesional en psicopedagogía». Acceso: 6 de agosto, 2020. https://www.uca.ac.cr/carreras/maestria-profesional-en-psicologia/

Universidad Latina. «Maestría en psicopedagogía». Acceso: 6 de agosto, 2020. https://ulatina.ac.cr/wpcontent/uploads/2017/12/Programa_Psicopedagog\%C3\%ADa_SP_baja.pdf

Universidad La Salle. «Maestría en psicopedagogía». Acceso: 7 de agosto, 2020. https://www.ulasalle.ac.cr/posgrados/

Universidad Latinoamericana de Ciencia y Tecnología, ULACIT. «Perfil ocupacional». Acceso: 7 de agosto, 2020. http://www.ulacit.ac.cr/carreras/seccion/perfil.php?career=6\&grade_id=5\& $\mathrm{id}=106$ 